\title{
The Study on the Governmental Tactics of Persuasion of Network
}

\author{
Public Sentiment
}

\author{
Zhang $\mathbf{Y i}^{1}$, Zhang Yinzhu' ${ }^{2}$ \\ ${ }^{1}$ School of Public Management (SPM), Yunnan University of Finance and Economics; ${ }^{2}$ Law School of \\ Yunnan University of Finance and Economics, Kunming, \\ P.R.China 650221
}

\begin{abstract}
As the intensive reflection on the internet of thoughts and emotions of netizens and interests of the masses, network public sentiment has always been the focus of attention and discussion of netizens, mirroring the thoughts and ideas of netizens in a certain era. There have been some limits in the hot-spot of network public sentiment because internet may serve as the communication channels for the harmful information and discordant sounds in spite of the convenience it offers to the freedom of opinion expression and participation in the discussion of public affairs of social individuals. Consequently, how to efficiently guide the hot-spot of the network public sentiment so as to make the harmonious network environment play an important role in the construction of a harmonious socialist society has been a major task for the information work of network public sentiment.
\end{abstract}

Keywords: Government, Network Public Sentiment, Persuasion

\section{Introduction}

Due to the solid influence of the network public sentiment on the social management and informational control of the government, efficient dealing with the network public sentiment has become the manifestation of the governmental office capacity. The government has to strengthen the mechanism construction of the network public sentiment in order to efficiently control the network public sentiment. It is practically significant for the government, as the principal part of public management, to focus on the key-points and core issues of practical works, conform to the developing tendency of network communication and correctly guide the network public opinion, adapting to the development of new situation, establishing the macro-vision and facing squarely the internet so as to implement scientific development vision, achieve healthy-rapid development of economical society and maintain the social stability and calmness of public feeling.

\section{Equal Dialogue: the Basic Notion of Governmental Persuasion of the Network Public Sentiment.}

The diversification and acutance of social public opinion in transitional period aggravate the pressure on the governmental guidance of public opinion. Suppressing the network public opinion by means of administrative control is far from feasible in political, legal and technical aspects in our democratic state in which freedom of speech is the basic right of every citizen entitled by the Constitution. It is wise and acceptable for the government to improve the occasion by the way of equal dialogue and promote the mutual understanding and consensus between the government and the public in order to guide the network public opinion into the direction of public interests.

Persuasion of public sentiment in essence is the course in which the public attitude is changed through various media communications and values which are made to be acknowledged by the public in accordance with public interests. It is impossible to force network public opinion, so the role which indoctrinization plays is limiting if the hearts and minds of netizens failed to be captured and echoed. To build up equal dialogues between government and netizens, works in four aspects should be enforced as following: (1) treat netizens sincerely. The trust between the government and the public can not be established and the rational consultation and dialogue on public affairs can not be carried out let alone reach the consensus and guide the public opinion unless the government faces the public sincerely, addresses the problems frankly, 
directly and openly and give the public the right of learning the truth in time. (2) The government should voice the public interests. As the trust institution of the public, the government should take the stand of the public and safeguard the public interests on the basis of public interests. (3) The government should communicate with the netizens equally. In the association on the basis of dialogue, the government should persist in the equal interactivity between the principal parts to accomplish understanding of life in common by equal discussion instead of deciding the host-guest parts and manipulating other participants by language threat or lure. The "discourse of power" leads to nothing but estrangement. (4) Observe the principle of open-dialogue. The government should be open to different opinions and make public the content of the dialogues in order to be helpful to form the consensus. All should be discussed openly except as otherwise provided by law and the refusal of discussion with the excuse of state secret is not acceptable. Otherwise, estrangement between the government and the public will be deepened.

\section{The Principle of Persuasion of Network Public Sentiment}

The crisis of governmental public opinion in network society is a new type one in the new situation of informationalization. As far as experience is concerned, we have less successful cases to learn and it is difficult to form a mature system of handling the public opinion crisis in a short time. Because of that, some basic governing methods are of great importance and can offer some significant help for our dealing with the crisis. The simple analysis of some basic principles of governmental persuasion of network public sentiment will be made as following:

\subsection{The Principle of Time First}

Presently, "the principle of time first", which is familiar to us, appears frequently on various newspapers and magazines. In the award report of the 18th session of China News Award and the 9th session of the Yangtze river TaoFen award, comrade Li Changchun also emphasized that "we should abide by the principle of time first, accuracy and transparency and release the authoritative information as early as possible so as to meet the informational demand of people and respond to the concerns of the society. We should also strive to seize the opportunity, win the discourse power and possess the initiative, firmly capturing the commanding elevation of public opinion persuasion." What is the first time of "public sentiment persuasion"? In the context of "first time" of public sentiment persuasion, the standard judging first time refers to the fast reaction, possession of speech initiative of public sentiment persuasion and initiative setting of the agenda of media and public instead of being driven passively by media and public agenda. To master the first time of persuasion of network public sentiment, firstly, it is favorable to pay attention to the timeliness, namely, it is better to release accurate information within 6 hours (at least 24 hours) after the incident. Secondly, from the prospect of coexistence, mutual boost and mutual confliction of network media and traditional media, it is necessary to pay close attention to the network public sentiment, make analysis, study and judgment in time and release accurate information betimes, taking initiative to guide the public sentiment before the following-up of the traditional media. Meanwhile, it is necessary to pay close attention to the hot report of traditional media which will be immediately copied by the network and release authoritative information in time to avoid the sensationalization on the internet.

\subsection{The Principle of Responsibility}

Daring to shoulder the responsibility is the top principle for the government facing the network public opinion crisis, especially at the beginning of the public opinion crisis. As the most active reflection of public feelings, network public opinion actually bears the requirement and expectation of the public to the government regardless of its form. The public opinion, whether it is praise or criticism, expresses the public expectation that the government shoulders the responsibility consciously. In the democratic period, the responsibility is the most important standard of the public to measure and evaluate the government. In the breaking-up of public opinion crisis, all the public targets the governmental responsibility. If the government is not bold enough to take the responsibility or even evades and conceal the truth, the media and the public will feel it necessary to make a thorough investigation. The curiosity about the incident it self will drive the wide spreading of all the information at a faster speed, which will attract more people into the comment about it. On the contrary, if the government, especially the main leaders of the government takes the responsibility first, the public will be convinced that the government is honest enough to address the problem responsibly. Then, some irrational speech will decrease sharply, which will not only appease the aggravating incident but also relieve the pressure of public opinion on government. 


\subsection{The Principle of Information Disclosure}

Unlike the traditional society where the information is released by the mainstream media and the general public has less freedom of choice as the receiver of the information, the network society serves as an information supermarket where the general public are not merely the receiver of the information but also have more freedom and choice, being able to select and filter the information according to their demand and interests. It is foolish to withhold the information which may lead to the opposite effect because it is difficult to suppress the information dissemination in technique which spreads in many ways and is very convenient in network society. When the negative public opinion appears, the government has no choice but frankly releases the information to meet and safeguard the demand of learning the truth of the public. To prevent the erroneous reports and rumors from circulating so as to dissolve the public resentments, the government should disclose the information and make the public the truth of the fact, the historical side and the way of handling at the beginning of the incident to relieve the pressure of public opinion. Only by this, the favorable condition can be created for setting the agenda again, guiding the public opinion and solving the crisis.

\subsection{The Principle of Sincere Dialogue}

Facing the negative pressure of one-sided public opinion, it is wise for the government to reconstruct the new mechanism of dialogue among the government, the public and the media, to reach the consensus through sincere dialogue and to rebuild the understanding and acknowledgement of the media and the public. The solution of group polarization effect spread by network information can only be achieved by sincere dialogue to make different opinions and advises expressed equally and share different experience so as to disclose a clear truth to the public and reach the consensus. To deal with the public opinion crisis, the only strategical choice for the government is to participate the discuss of the incident free from the bureaucratic monologue in the honest, sincere, equal and open ways and make different people talk to each other by means of sincere consultation of interactivity between different parts to guide the public opinion to the favorable direction, if the government wants to establish significant public discuss by which the pressure of irrational and negative public opinion will be relieved.

\subsection{The Principle of Capturing the Speech Power and Reset the Topic of Public Opinion}

The network society has different power structure from the traditional society. In the traditional society, as the only owner of power, the government can implement the social order and decide the direction of public opinion with its power. In these aspects, the government has no rivals to compete with. The network topology is in the form of plane and there are many communication channels between every nodes. It is impossible for any informational nodes in the network to become the only monopolist of information due to the crisscross, fast and convenient informational transfer of "decentration". In times of network, the government has no longer the position of informational monopoly and the power of speech monopoly of the government has been deconstructed by the equal and interactive dialogue. Fighting for the speech power will be endless because of the decentralized speech power in the network society. On the internet, those who takes initiative on information will win the speech power and be the leader of opinion. However, the fight for the speech power will be everlasting while leader of opinion will not. Therefore, it is of high importance for the solving the crisis of public opinion to positively strive for the speech power and reset the agenda of public opinion.

\section{The Governmental Persuasion Measures of Public Sentiment}

\subsection{We should listen attentively to the “Network Public Opinion" to promote the Guiding Ability of Network Public Opinion}

Cadres and leaders should fully understand that internet has become the key expression channel of the public attitudes and adapt to this change. Some believes that "the grass root organization, the ideological work and the police are kept away from the hot-spot on the internet”. From the aspect of the way and the channel of the power execution, it is hard to contact the numerous participants in the hot event on the internet face-to-face. But if the cadres and leaders can change their minds to regard the network as the forefront of research and working place of doing the mass work and maintaining the social stability and positively respond to the network speech with the equal, respecting and sincere attitude and without any haughty manner, the network advantages will be made full use and disadvantages will be avoided to transmit 
the voice of the government and the Party. After the happening of the public emergency, the cadres and leaders should not only properly handle the emergency in reality but also make a good job of network monitoring and alarming. They should learn the public sentiment and developing tendency of network public sentiment, give the respond in an official stand and governmental voice into the public informational system as early as possible to block the rumor and clarify the hearsay, appease the radical public resentment with authority by guiding the public to tell the clear difference between the truth and the rumor, make the objective judgment and take rational actions to reach the crossover point so as to solve the problem. In the network era and world, the cadres and leaders can be neither blind nor mute and arrogant. Only the initiative attitude can bring the initiative in public opinion and disposition of events.

\subsection{We should maintain the Right Guidance of Public Opinion, Overwhelming the Negative}

Public opinion with the positive one. Internet has become the new forefront and new battle field of public opinion. The propaganda on the internet should keep in line with the correct direction of public opinion, construct positive public opinion of mainstream, take initiative to "set the topic of public opinion” and occupy the living-space of various unharmious sounds with positive propaganda to get rid of all the bad influence of mistakes and reactionary opinions. Especially for some serious events, we must take the prudent attitude, try our best to give the objective, real, comprehensive and fair report and avoid partiality, information asymmetry, social misleading and trouble. We should differentiate some public opinions of different natures: the first one is the normal concern about the major events and hot-issues and the reasonable request of solving problems in the reform of the common people; the second one is the negative and radical speech about the dark side of the society, the corruption cases and emergency; the third one is the reactionary speech disseminated by the enemy inside and outside and the hostile attack through various hot and different problems and emergency. For the first one, we should strengthen the persuasion and guidance by positive propaganda and publicize targetedly the relevant policies of the Party's central Committee and State Council to appease the contradiction and the public resentment. For the second one, we should immediately clarify the distorted truth and skillfully guide the radical speech. For the third one, we should engage in the fight of public opinion to refute powerfully the aggressive speech and the rumor made by the enemy in time. Especially in emergency and group event, we should discover betimes the network instigating speech of the enemy inside and outside and unmask their sinister and ulterior purpose.

\subsection{We should erect the idea of equal Dialogue and Enhance the Effect of Political}

Communication in the network space. The development of network technology will give powerful support of material and techniques to the development of democratic politics. In the near future, collecting advices on administration from internet may become the major way of governance. Therefore, it is no doubt that we should introduce the network techniques and media into the political life. Furthermore, we should make the best use of these material and techniques to promote the art of governance. For us, works in the following aspects should be done well, if we want to enhance the effect of political communication in network-space.

Firstly, the mechanism of public attitude collecting and responding should be established and improved to form a better interactive order between the government and the society. The fast and wide-spreading communication of network will broaden the impact of group events, causing the unpredictable social turbulence. Therefore, relevant governmental departments should strengthen the informational supervision on the internet and keep track of the development of public opinion. First of all, the monitoring software of network public sentiment should be developed. Then, the 24-hour monitor of network information should be made by specialized personnel designated to control the tendency of network public sentiment and detect the information which is harmful to the social stability; the analysis of public sentiment information which is informative, thoughtful and deep should be strengthened by means of collecting, sorting out and summing up the scattering and elementary information; the occurrence, development and tendency of group events based on the network should be carefully studied to provide party and government departments with the measures and advices on the preventing and addressing the group events.

Secondly, centering on the legal construction of socialism, the cultural construction of network should be strengthened and the network ethics should be purified to create healthy environment of network communication. Nothing can be accomplished without norms or standards and the anonymity and openness of the network impairs the veracity and feasibility of the network information, triggering a great deal of rumor and radical 
speech, which entails the power management of the government. The proper control on the network should be made and the legislation is an important way for the governmental control. The state has promulgated a series of laws and regulations such as Decision on Safeguarding Computer Network Security, Administration of Internet Information Services Procedures, Interim Provisions on the Administration of news publication of the websites and Administration of Internet Electronic Messaging Services Provisions. Party and government institutions should ensure the enforcement of these laws and regulations with powerful administrative measures, tighten the access permission of network media, strengthen the supervision and control on the network media and standardize the network space.

Thirdly, centered on the social cultivation of the citizens and focusing on the promoting the public rationality, the political expressive ability and rational negotiation of the citizens should be improved. Internet is just the platform of information exchange and the start and development of network public sentiment are mainly droved by netizens who are the main body on the internet. To eradicate the false network information and radical speech, the network ethical education of netizens should be strengthened and the advanced culture of socialism and the network concept of honor and disgrace should be publicized to make the netizens start from their own behavior to surf on the internet civically and healthily so as to achieve the self-discipline of the netizens. Only by means of democratic political experience to press ahead the education of citizens, improve their public rational spirit, foster democratic and rational citizens can the network political culture get the development in order and effect of political communication in net-space keep being promoted.

Fourthly, by means of conscious policy support and bridging the "informational gap" with endeavor, more chances should be offered to the disadvantaged groups to obtain information on the internet and express the interests demand. In the policy selection, the western area, backward area, villages and no-center cities should get more support to strengthen the ability and condition of disadvantaged areas and groups to obtain the information with network, to guarantee their right to learn the truth, participate and express so as to improve their ability in policy game. Additionally, the network charges should be reduced to ensure that no one of the disadvantaged groups will be kept away from the internet and the their ability of getting information with internet and expressing the interests demand will not be harmed because of the economic reason. With the bridging "knowledge gap", the network technology can benefit the ability of every citizen of expressing their interests demand and equally accessing the interests game. Thus, the network political action can reflect well the public will.

\subsection{We should comply with the Development Tendency of Network Communication and Improve}

The skills of network communication. Without the relevant action in time, it is impossible to change the mind, choose the correct tactics and solve the problems of network public sentiment persuasion. "Network skills", which the Party and government institutions, enterprises and individuals skillfully and initiatively use in time through such new media means of internet as official and media websites, message boards of netizens and official and individual blogs to release information and communicate opinion, is to deal with public sentiment. Being familiar with network propaganda, internet techniques and network public sentiment persuasion and building system and mechanism of "network in politics" are beneficial for heeding to the public will, appeasing the public resentment, dissolving the confliction and promoting harmony as well as maintaining the government credit and improving the effect of handling the public sentiment. In the situation of complicated and sharp network public sentiment in which the public opinion spreads at the speed of light, the substantial achievement can only be accomplished with the initiative, swift and innovative action.

Firstly, the standing network platform of interactivity between the government and the public should be built. Without an unobstructed channel for the communication of the government and the public, relevant public opinion will concentrate and spread immediately on the internet once the emergency occurs. If the opinions and complains of netizens can not effectively transmit in time to the governmental administrative departments, the negative information will keep swelling, which will lead to more damages. Consequently, building the network interactive channel with the netizens by the government in the environment of network media to control the public sentiment is an indispensible measure. On one hand, further and specific understand of various opinions and questions of the netizens will be made to transmit the governmental voice and guide public opinion in well-directed pressing brief. On the other hand, the effective operation of interactive channel can meet the demand of participating in the administration and discussion 
of state affairs of netizens. With the economic development, educational promotion and concept improvement, the master awareness of the public is increasingly strengthened, which made their desire to participate in the administration and discussion of state affairs become very strong. Therefore, one of the major measures of the government to guarantee the public right to participate in the administration and discussion of state affairs is establishing the network interactive platform of administrative information which should also be continuously improved and institutionalized. The institution of that should include unobstructed channel, netizen reflection and department respond to ensure the smoothness and feedback of the channel instead of being a mere formality.

Secondly, the positive guiding force of the "public opinion leader" should be strengthened. According to the law of communication, the attitude of audience is always influenced by the "public opinion leader". When the emergency occurs, a great deal of information concentrates on the internet. As netizens are unable to tell the truth of the fact form the fake, they usually make their judgment according to the authoritative voice of the network public opinion. The authoritative "public opinion leaders" on the internet include network spokesmen of the government, network commentators, some of experts, BBS moderators, famous bloggers and so on. It is the main task of current persuasion work of network public opinion to reinforce the positive guiding force of the public opinion leaders so as to achieve the virtuous circle of the network public opinion under the guidance of the network opinion leaders. For this purpose, the network spokesmen and the team of network commentators of the government should be cultivated and the collaboration of the non-governmental leaders of public opinion is necessary to scientifically guide the direction of public opinion. The leaders of opinion should also be encouraged to report the truth and transmit the governmental voice to serve the guidance of network public opinion in order to achieve the effect of "guide the netizens by themselves".

\section{Conclusions}

The report of 17th National Congress of the CPC clearly put forward that "the construction and management of network culture should be strengthened and better network environment should be built”. Due to the complicated situation of network ideological struggle, we should attach great importance to network, take the initiative to guide the public opinion, possess the speech power and form the joint force, promoting the guiding ability of network public opinion hot-spot and grasping the initiative of public sentiment persuasion. Otherwise, the control of public opinion will be lost to impact the stability of society.

\section{Acknowledge}

The Research on Governmental Persuasion of Network Public Sentiment (NO.JCZX201207), the planning programming of philosophy and social sciences in Yunnan.

\section{Reference}

[1] Fan Peng, Wei Shengwen, Wei Qi. Analysis and Forecast on Public Opinion of Gansu from 2006 to 2007[M]. Gansu: Press of Gansu People, 2006. (In Chinese)

[2] The Bureau of the Public Sentiment Information of Publicity Department of the CPC. The Survey of the Information Work of Public Sentiment[M]. Beijing: Press of Study, 2006. (In Chinese)

[3] A Website in Xiamen Was Investigated and Punished Due to its Dissemination of the Rumor of “Sepat” [N]. People’s Daily, 2007, August, 22nd

[4] The Bureau of the Public Sentiment Information of Publicity Department of the CPC. The Study of the Collection and analysis Mechanism of Public Sentiment Information[M]. Beijing: Press of Study, 2006. (In Chinese)

[5] Zeng Baode. Some Ideas on Establishing Positive Majority of Network Public Opinion[N]. China News, 2007, August, 15th

[6] Li Qiongyao. Present Situation and Guidance of Network Public Opinion[J] Journal of Hunan Administrative College, 2006, (2) 